\title{
Modelling the Chemical Evolution
}

\section{Gerhard Hensler and Simone Recchi}

Institute of Astronomy, University of Vienna, Tuerkenschanzstr. 17, A-1180 Vienna, Austria email: gerhard.hensler@univie.ac.at, simone.recchi@univie.ac.at

\begin{abstract}
Advanced observational facilities allow to trace back the chemical evolution of the Universe, on the one hand, from local objects of different ages and, secondly, by direct observations of redshifted objects. The chemical enrichment serves as one of the cornerstones of cosmological evolution. In order to understand this chemical evolution in morphologically different astrophysical objects models are constructed based on analytical descriptions or numerical methods. For the comparison of their chemical issues, as there are element abundances, gradients, and ratios, with observations not only the present-day values are used but also their temporal evolution from the first era of metal enrichment. Here we will provide some insight into basics of chemical evolution models, highlight advancements, and discuss a few applications.
\end{abstract}

Keywords. galaxies: abundances, galaxies: evolution, Galaxy: abundances, Galaxy: evolution, ISM: abundances, stars: abundances

\section{Introduction}

The evolution of the Universe is clearly manifested by its structure formation and the consumption of gas to star formation, the latter leading to the enrichment of chemical elements heavier than those stemming from the primordial nuclesynthesis. Knowing four facts, the star-formation (SF) rate at any time and its integral over the Hubble time, the initial stellar mass function (IMF), the production and yield of each element for each particular stellar mass, in each stage of the stellar life, respectively, would well define the interstellar enrichment and with some SF delay also the stellar, so that an observational trace-back of the chemical evolution (CE) of the Universe would allow to derive one of these parameters definitely and for different specific structures.

Assuming that a structure like e.g. a galaxy in total or also any galactic region under consideration behaves like an isolated volume, called a closed box, the longer-living lowmass stars lead to increase the stellar mass fraction continuously as well as the stellar remnants' lock-up mass while as a consequence of SF the gas fraction diminishes. The basic set of analytical equations for the chemical evolution of galaxies has been formulated by numerous authors (e.g. Talbot \& Arnett (1971), Pagel \& Patchett (1975), Tinsley (1980), Pagel (1997) and a lot more since then: see references in the recent papers by Prantzos (2008a) and Recchi et al. (2008)). In this simple model a temporal relation for the metallicity as $Z_{i}(t)=y_{i}[-\ln (\mu)]$ follows where $\mu=M_{g}(t) / M_{(g, 0)}$ is the temporal gas mass fraction and $y_{i}$ the yield of element i, i.e. the metallicity release per stellar population (see left panel of fig. 1).

Unfortunately, nature is not as simple and our present knowledge has a lot of uncertainties and even gaps because of models that are reasonably and necessarily much simpler than nature and some processes are not yet well understood. A strong simplification is implied to the analytical solution by the lifetimes of the stellar factories and thus the various delay times of specific element releases. The discrepancy of the so-called instantaneous recycling approximation becomes obvious after almost 2-3 Gyrs as demonstrated in fig. 12 of Prantzos (2008a). A trace-back of element ratios through long-living 

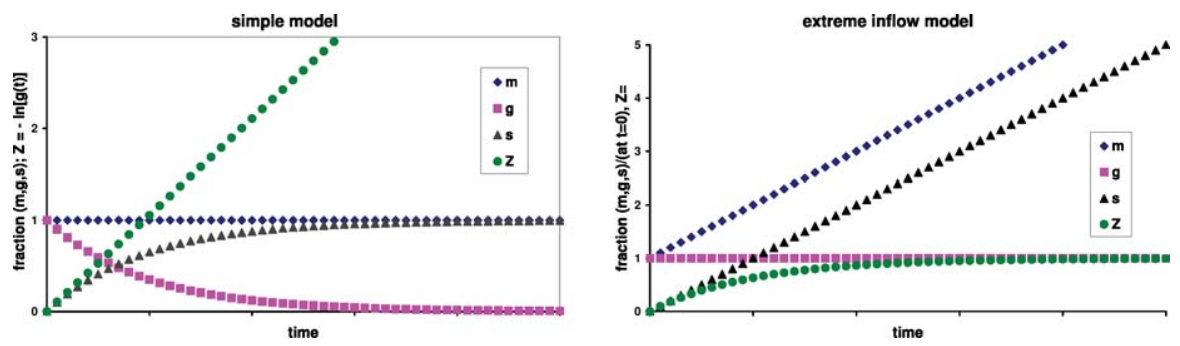

Figure 1. Evolution in arbitrary time units of an element $Z_{i}(t)$ (green dots), of total mass $\mathrm{m}$ (blue rhombi), gas mass fractions g (red squares), and stellar mass fraction s (black triangles) with respect to $\mathrm{t}=0$ for a closed-box model (left) and for the extreme inflow of primordial gas (right) necessary to get the gas mass g constant.

stars is clearly representing this delay, the most prominent signature being e.g. in the $[\alpha / \mathrm{Fe}]-[\mathrm{Fe} / \mathrm{H}]$ trend of the solar vicinity.

Another simplification can be relaxed by advancing the closed box to open boundaries and allowing for gas infall or outflow. Fig. 1 (right panel) demonstrates the extreme case of gas infall which is required to balance the gas comsumption. By this, also the stellar $\mathrm{Z}$ enhancement is reduced by dilution with primordial gas. As a further limitation the released gas is assumed to mix instantaneously with the local environment.

Another effect also enters into the element distribution, namely, the existence of different gas phases produced by means of various heating processes and the splitting of stellar metal injecta to these phases. While the SF is limited to the cool gas alone, cooling timescales of dilute hot gas are extremely long, by this, allowing to refrain characteristic elements from their immediate incorporation into newly formed stars. The ignorance concerning such small-scale mixing timescales between the different gas phases until now allows only an order-of-magnitude approach for chemical models. Mixing processes are not only caused by diffusion in an otherwise static gas but triggered by dynamical effects. This means that last but not least basic ingredients to understand and to model the CE is gasdynamics.

Despite of the enormous activities all over the field of CE but because of limited space and time in this paper, we cannot cover all aspects and objects under investigation of CE and also not refer to the whole bunch of literature, but have to pick out a few papers as representative and review the present state of modelling the $\mathrm{CE}$ of some cosmic structures only from the early universe to the local. We therefore apologize for the reasonable incompleteness. Starting from the solar vicinity the paper focusses on some hot topics as e.g. the galactic halo and its dwarf spheroidal (dSphs) satellite system finishing with dwarf gas-rich galaxies.

Since present-day chemical models are preferentially performed by numerical simulations a further aspect on the quality and reliability of models should focus on the applied numerical methods. Since it is not the aim of this review to also address and discuss the validity of numerical methods of galaxy evolution, the interested reader is referred to a dedicated recent review by Hensler (2008).

\section{The Milky Way components}

That our Milky Way has experienced such a CE became already obviuos from the findings by Sandage \& Fouts (1987) of the metallicity increase from halo to thin disk stars. Although large-scale dynamical effects during the formation and evolution of galaxies, as e.g. gas accumulation by infall, galactic winds, etc., are proven by observations, models of 
the galactic CE are mostly executed for the different galactic components in separation only and to some extent implying dynamical processes thru analytical terms, not selfconsistently but instead tuning them corresponding to a best fit of the observations.

\subsection{The Milky Way disk}

One of the problems within the solar neighbourhood realized at earliest already by Beatrice Tinsley (1974) was the so-called G-dwarf problem, the lack of low-metallicity G dwarfs. Although this problem can be simply solved by exponentially declining infall (but also outflow or both), Pagel (1987) has already emphasized that additional deviations from a constant slope in the $\ln (\mu)-Z$ diagram, the yield $y$, point towards dynamical influences. Moreover, Prantzos (2008a) (his fig. 14) shows that a metal pre-enrichment leads to a better fit to the stellar $Z-\ln (\mu)$ distribution.

Therefore, non-dynamical CE models have been conducted with a temporal and radial function of a gas infall rate (see e.g. Chiappini et al. 1997, Portinari \& Chiosi 1999). Because also the SF rate is related to the gas surface density $\Sigma_{g}$ as $\Psi(r, t) \propto \nu \Sigma_{g}^{k}(r, t)$, where $\nu$ represents a SF efficiency, the infall steers the SF. The exponent $k$ can be set to unity or adapted to the original Kennicutt (1989) relation 1.4. Additional factors are also included, as e.g. the total mass density (see e.g. Chiappini et al. 1997, eq. 4).

The most cited model of this kind by Chiappini et al. 1997 can fit the G-dwarf problem at best by two infall episodes, a short one with 1 Gyrs timescale only and a long one of 8 Gyrs. Astrophysically speaking this so-called two-infall model resembles nothing exceptional or unexpected than a short initial collapse phase and the general gas assembly with long-term decreasing infall rate. Nevertheless, also the SF efficiency as another free parameter is changed between both infall eposides. Depending on the stellar sample used for the G-dwarf problem the models fit more or less (Chiappini et al. 1997), but a quantitative comparison of the model abundances at present-day with solar is desillusionary and not well surportive for the model since it provides not more than a qualitative tendency. The same is discernible for the stellar age-metallicity relation (AMR) in Chiappini et al. 1997.

On short timescales, however, the gas accretion rate should vary locally and affect the SF (Pflamm-Altenburg \& Hensler 2010) and the CE (Hensler et al. 2004a) as well as the abundances (Koeppen \& Hensler 2005) what was also invoked from observations of the solar neighbourhood by Knauth et al. (2006).

How uncertain the deduction of relations can be and to some extent biased by the sample taken for studies can also be documented by a comparison of the original AMR of stars found by Twarog (1980) with that derived by Nordstroem et al. (2004) from a well-defined and complete sample of solar vicinity stars. While the traditional results revealed the expected tendency but with very strong early enrichment, already Tadross (2003) did not find any relation at all from open cluster analyses, while Nordstroem et al. (2004) data yield a trend. Showing a large hardly understandable scatter $\Delta[\mathrm{Fe} / \mathrm{H}]$ by almost 0.5 , even for the average AMR tendency a CE model is hardly constructable without any metal pre-enrichment.

The evolution of radial abundance gradients is reasonable, but values, ranges, and the temporal behaviour are also uncertain (see Maciel \& Costa, this volume). Galactic abundance gradients that are measured from HiI regions as usually by the standard method of collisionally excited emission line (CEL, Shaver et al. 1983) or recently used recombination lines (RL) in the infrared (Esteban et al. 2005) can only yield the presentday abundances in the ISM. Over years of determinations the slope has not only been reduced, e.g. for $\log (\mathrm{O} / \mathrm{H}$ ) from -0.8 per kpc (Shaver et al. 1983) to -0.44 (Esteban et al. 2005), but also a flattening of the gradients in the innermost radii and also outside the 
solar circle (Vilchez \& Esteban 1996) became available from RLs. From massive stars one can derive the same: the chemical abundances of the ISM from which they are born most recently.

While Maciel et al. (2003) could derive a temporal flattening of the oxygen gradient over the past 9 Gyrs from -0.11 to -0.06 dex/kpc unsing Planetary Nebulae, from stars of different ages Nordstroem et al. (2004) could even attribute a positive slope to disk stars older than 10 Gyrs, and again a slight flattening for younger stars from almost -0.1 dex/kpc to -0.077 since 1.5 Gyrs.

While the present-day abundance gradients from HiI regions hold for all the determined elements, i.e. $\mathrm{C}, \mathrm{N}, \mathrm{O}$, and $\alpha$ elements, their different radial slopes need explanation. Interestingly, the abundance ratio $\mathrm{C} / \mathrm{O}$ shows also a radial decline what means that $\mathrm{C}$ is more abundant in O-rich regions. As explanation Carigi et al. (2005) proposed that this behaviour is caused by a metal-dependent $\mathrm{C}$ yield. On the other hand, also the $\mathrm{O}$ yield is metal dependent in the sense that at lower metallicities a weaker wind by massive stars allow a longer shell-burning production of O (Maeder 1992). Cescutti et al. (2009) modelled the chemical evolution of bulge and disk stars with metal-dependent yields by Meynet \& Maeder (2002b) and could by this also reproduce the enhancement of $\mathrm{C} / \mathrm{O}$ in metal-rich regions like the bulge.
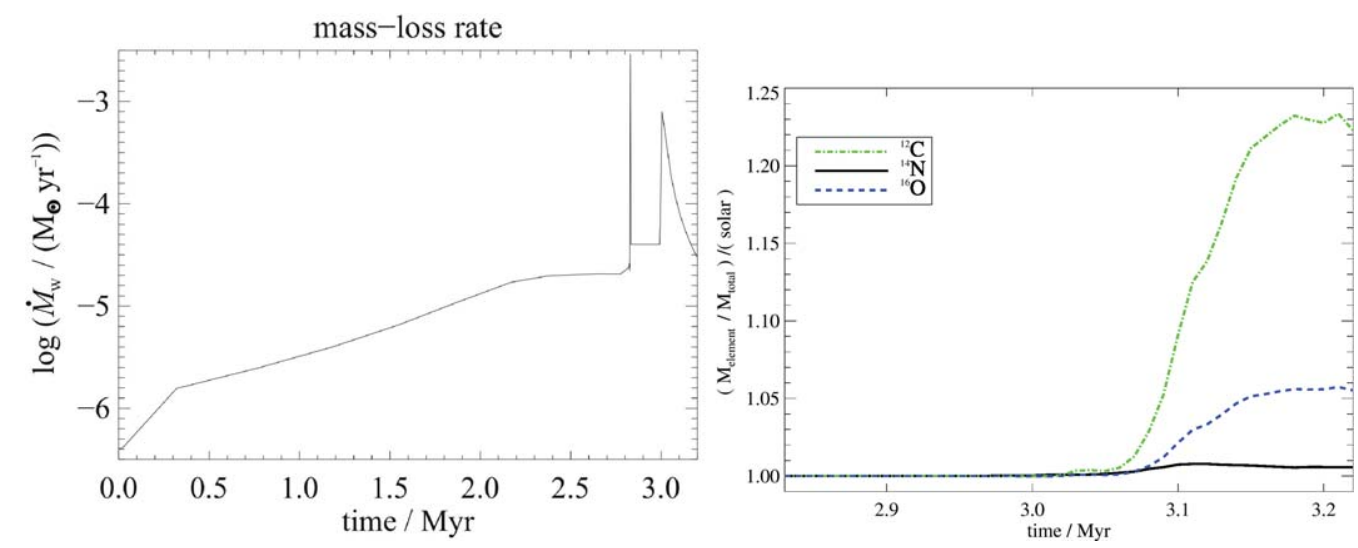

Figure 2. Evolution of a $85 \mathrm{M}_{\odot}$ star until its explosion as supernova typeII. Left panel: Wind mass loss adapted to evolutionary models by Schaller et al. (1992). Right panel: Time-dependent abundances of ${ }^{12} \mathrm{C},{ }^{14} \mathrm{~N}$, and ${ }^{16} \mathrm{O}$ in the warm $\mathrm{H}$ II region gas after the onset of the WN phase at 2.83 Myrs. See Kroeger et al. (2006).

For the determination of $\mathrm{C}$ and $\mathrm{O}$ in HII regions, another effect has to be studied seriously: self-enrichment by Wolf-Rayet stars (Kroeger et al. 2006). At the end of its lifetime at $t=3.22 \mathrm{Myr}$ a $85 \mathrm{M}_{\odot}$ star has supplied $0.28 \mathrm{M}_{\odot}$ of ${ }^{14} \mathrm{~N}, 13.76 \mathrm{M}_{\odot}$ of ${ }^{12} \mathrm{C}$, and $11.12 \mathrm{M}_{\odot}$ of ${ }^{16} \mathrm{O}$, which are contained in the combined stellar wind bubble/Hir region. Since $\mathrm{N}$ was released at first in the WN stage it increases slightly in this period and is thereafter diluted by the N-poor gas feed. These facts are discernible in the hot gas by a first rise and a subsequent decrease of the $\mathrm{N}$ abundance (Kroeger et al. 2006) after the transition to the WC stage when $\mathrm{C}$ and $\mathrm{O}$ are released. The $\mathrm{C}$ content in the hot gas increases steeply and reaches an overabundance of 38 times solar while the enrichment with $\mathrm{O}$ is weaker. Due to turbulent mixing of the hot gas with the photo-evaporated shell, the warm HII gas is also enriched but at most for $\mathrm{C}$ by not more than a factor of 1.22 (Fig. 2, right panel). Since the stellar mass range of the Wolf-Rayet phase increases with 
metallicity, the negative slope of the $\mathrm{C} / \mathrm{O}$ ratio within the galactic disk can be attributed to this effect.

In a recent study Colavitti et al. (2009) found that the chemical properties of the galactic disk are at best reproduced by models with an infall law derived from cosmological but purely Dark Matter simulations. In reality, the disk settling timescale is not a simple stellar dynamical one but inherently determined by its thermal and turbulent energy budget causing a delayed thin-disk formation following the thick disk (Burkert, Truran, \& Hensler 1992).

\subsection{Multi-zone models}

As already implied as advancement of simple CE models one can even account for gas exchanges through the boundaries of limited regions under consideration by simply adding a corresponding time-dependent (source or sink) term to the gas-mass and metallity equations. This was e.g. done in several above-mentioned models with gas infall to the galactic disk. Nonetheless, no simple model exists that also accounts for outflow from the disk. A first step was the exploration of the effect of the galactic fountain model on radial metallicity gradients by Spitoni et al. (2009). The idea is, that the disk gas pushed-up by supernovae ( $\mathrm{SNe}$ ) and redistributed while falling back on ballistic paths has to mix with the disk gas within the site of impinge and affects the abundance gradient of the galactic disk. As general results they found that neither the ballistic delay nor the cooling delay of this fountain-driven mixing process alter the metallicity and the radial oxygen gradient.

As well as in this approach also e.g. for infall models, authors preferably avoid the complexity of a proper hydrodynamical treatment combined with the chemical and thermal descriptions by means of an analytical ansatz. From gas infall and expected radial gas flows, the above mentioned models mimic the first process by radius-dependent functions, but drop the latter effect. In order to differentiate between radial disk regions and to follow radial flows, multi-zone models were developed e.g. that devide the galactic disk into rings and allow for a radial gas flux, are artificially parametrized due to positive flows characterizing the inside-out evolution of the disk (Portinari \& Chiosi 2000) or are consistently determined by the gas pressure gradient.

Different approaches were performed by coupling structural parts of the Milky Way, e.g. halo and disk, and dealing with mass exchange between the regions (Ferrini et al. 1992, 1994). By fine-tuning not only the coefficients for mass exchange arbitrarily, but also further parameters, as e.g. SF efficiency, etc., rough quantitative agreements with gas and stellar mass, abundances, and their radial gradients in the disk could be achieved.

\subsection{Chemo-dynamics}

For all these simple-type CE models it is inherent that their results in comparison with observational facts can provide a first insight into amount and timescales on which nonlocal effects have played a role during the evolution. Nonetheless, they are all devoid of self-consistency according to the acting processes and the resulting dynamics. Two major sources exist, driving the gas dynamics, namely, gravitation and stellar energy release. Plasmaphysical processes according to heating and cooling lead to different gas phases that have not only different energetics but also behave dynamically differently and interact by multiple processes as e.g. by drag forces, shock waves, and turbulence as well as energeticly e.g. by heat conduction. Moreover, this means that also SF and thermal state of the ISM have to be treated consistently.

A first-order approach into this direction is provided by multi-phase CE models (also implied in Ferrini et al. 1992, 1994) where, however, also the interaction terms in a set of equations are not self-consistently determined but arbitrarily applied. 
How crucial an appropriate representation of the ISM and its processes is, will be demonstrated in a few of the next sections. Two main ingredients can already be established here: 1) Cool/warm and hot gas phases are coupled energeticly by heat conduction, leading to a self-regulation of SF (Koeppen, Theis, \& Hensler 1998), local mixture of hot and cool gas and, by this, local metal enrichment of cool gas with SN elements. 2) The dynamical coupling of both gas phases by drag and mass loading due to evaporation hampers the outflow and enhances the cooling of the hot gas.

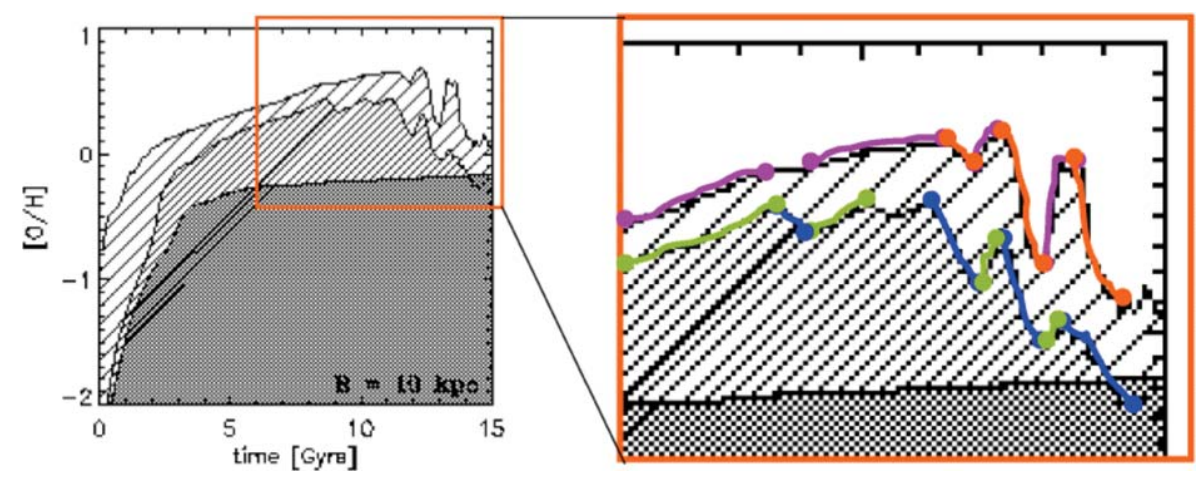

Figure 3. Metallicity evolution of three different components, cool/warm gas (CM, dark hatched), hot intercloud medium (ICM, light hatched), and stars (grey) on average of a Milky Way-type chemo-dynamical model (Samland, Hensler \& Theis 1997) at $10 \mathrm{kpc}$ galactocentric distance within the disk. The right panel is a cut-out of the left one demonstrating how the metallicity $\mathrm{Z}$ is affected by different processes: 1) Due to infall of primordial gas, $\mathrm{Z}_{C M}$ decreases. 2) $\mathrm{Z}_{I C M}$ increases due to supernova-expelled metals and is always higher than $\mathrm{Z}_{C M}$. 3) The $\mathrm{Z}_{I C M}$ decreases therefore, when CM evaporates by heat conduction. 4) Condensation of hot gas onto cool clouds increases their Z. The stellar Z represents an average over stars of all ages and is therefore smaller than $\mathrm{Z}_{C M}$. (from Samland 1994).

Up to now as the optimal grid code one can consider the further development of the chemo-dynamical scheme by Samland, Hensler \& Theis (1997) to 3D and with the stellar dynamics for the stars. In addition, a cosmologically growing DM halo is included into the simulations by Samland \& Gerhard (2003). These models contain all the crucial processes of SF self-regulation by stellar feedback, multi-phase ISM, and temporally resolved stellar components according to the chemo-dynamical prescription (Hensler 2003). They cannot only trace the formation and evolution of the disk galaxies' components but also of characteristic chemical abundances and are until now the best self-consistent evolutionary models of disk galaxies because the disk formation is included into the global temporal galaxy evolution.

\subsection{The Milky Way halo}

Until half a decade ago only poor knowledge on the element abundances of the Milky Way halo stars was available. With the wealth of new class 8-10m telescopes, spectroscopy of faint and distant halo stars has changed our picture fundamentally. While in the era of Sandage \& Fouts (1987) a halo metallicity $[\mathrm{Fe} / \mathrm{H}]$ not below -4 could be found and only a few below -3 , raising doubts on the first population of stars in the halo and their chemical fingerprints, recent detections of hyper metal-poor stars (Beers \& Christlieb 2005 ) in the galactic halo and their peculiar element abundances (see e.g. Frebel et al. 2005) opened a wide field of new activities, namely, modelling towards understanding the zero metallicity nucleosynthesis and studying the formation of the halo. This is necessary in order to decide between the two preferred formation scenarios of the Milky Way: the 
monolithic collapse model (Eggen et al. 1962) and the accretion model (Searle \& Zinn 1978). A general word of clarification of the two terms should be done here: Even if the accretion model has formed an inhomogeneous halo (Argast et al. 2000, Oey 2000) its mixing of infalling gas with stellar ejecta lead to a settling of more or less homogenised gas with low angular momentum into the bulge and with larger one into a disk. Due to the higher gas column density in the innermost disk due to its smaller area the mostly invoked inside-out evolution in the disk is quite plausible.

Although a dichotomy of halo stars, the inner halo vs. the outer halo (Carollo et al. 2005), makes a dissipative formation of the inner halo probable but invokes a dissipationless accretion process for the outer halo stars, it is still under debate whether part of the present-day dwarf spheroidal galaxies (dSphs) could have served as its building blocks. Although most of the dSphs have formed their stellar population in an early short, but active SF epoch, the chemical signatures of most of the dSph stars still alive are distinct from the stars of each kinematic component of the Milky Way (Venn et al. 2004).

Even the most recent investigation by Prantzos (2008b) which is sold as a clear proof that dSphs have served at least partly as building blocks of the Milky Way halo, lacks of consistency: SF history, baryonic mass content and metallicity have been assumed from the 4 most prominent present-day dSphs, and the accretion timescale was set to half a billion years only.

Since even the CE of the dSphs is not yet conclusively understood, in general, models have to be advanced for this morphological galaxy type.

\section{Dwarf galaxies}

\subsection{The Milky Way dwarf spheroidal satellite system}

Since the origin and the Dark Matter content of the galactic system of dSph satellites is basically questioned by some authors (Metz et al. 2009), general problems are: Can low-mass systems survive an intense SF epoch (as expected at the formation)?

From compilations by Dekel \& Woo (2003) and Grebel et al. (2003) dSphs follow a mass-metallicity relation. As an extreme case Hensler et al. (2004b) have performed spherical low-mass galaxies by means of chemo-dynamical simulations in order to study the galaxy survival, SF rates, gas loss, and (final) metallicity. They could demonstrate that due to the SF self-regulation only short but vehement initial SF epochs occur and lead to mass-dependent gas loss. Nonetheless, the dwarf galaxies (DGs) remain gravitationally bound with the further issue that more cool gas survives than it is observed, but it forms a halo around the visual body. Although the stellar energetic feedback is the driving mechanism to expel the gas, its effect is not as dramatic as obtained in semi-analytic models (Salvadori et al. 2008) and the amount of unbound mass is considerably lower. To get lost, this gas has to be stripped off additionally (Grebel et al. 2003) what probably happens because of the ram pressure of the galactic halo gas or of tidal stripping (Read et al. 2006). Otherwise it can bounce back to the DG and produce subsequent events, from a second SF epoch even up to SF oscillations. The external gas reservoir around the Scl dSph (Bouchard et al. 2003) might whitness this effect. While the models make some intermediate-age stars, most stars are made in less than 1 Gyr after formation. The dependence of stellar metallicity on the galaxy mass is a quite well confirmed observational relation.

Because of its simplifying one-dimensionality and the neglect of environment effects like e.g. tidal effects, external gas pressure, gas inflow, etc. more complex models are required. The fascinating wealth of data and their precision on stellar ages and kinematics, on their chemical abundances and tidal tails of dSphs (for most recent reviews 
see e.g. Koch 2009 and Tolstoy et al. 2009) have triggered numerous numerical models. Although all of them up to date are advanced by 3D hydrodynamics (see e.g. Marcolini et al. 2006 and Revaz et al. 2009), they still lack of the same aforementioned environmental agents. The most comprehensive paper by Revaz et al. 2009 e.g. simulates a bunch of DG models with the method of smooth-particle hydronamics (SPH), but again all of them in isolation. In their models mainly also sufficient gas mass is retained and can fuel further SF epochs if it would not be stripped of by ram pressure or tidal forces, as the authors mention. Those models that fit the presently best studied dSphs Fnx, Car, Scl, and Sex at best, are than assumed as test cases for further exploration. Although their results do not deviate too much from the further observational data, in addition to the already mentioned neglects, three further caveats must be mentioned: 1) If models are selected according to agreement with one or two observed structural parameters, it is not surprising if also other values would not deviate significantly. 2) The numerical mass resolution of the SPH particles is too low to allow quantitative issues of galactic winds, heating and cooling, etc. 3) Because of the single gas-phase description released metals are too rapidly mixed with the cool gas and the metal-enrichment happens too efficiently. Despite these facts, with appropriate initial conditions always models in agreement to observations can be found.

Although the advancement to a two-phase ISM by an SPH code is not trivial and inserts various numerical problems, but is not impossible (Berczik, Hensler, et al. 2003, Harfst, Theis \& Hensler 2006, Scannapieco et al. 2006), such treatment would be absolutely necessary in order to achieve reliable results. In addition the chemo-dynamical interaction proceeses must be implied.

\subsection{Dwarf Irregular Galaxies}

Dwarf irregular galaxies (dIrrs) are characterized by large gas fractions, often an ongoing $\mathrm{SF}$ and low metallicities. Until $\sim 10$ years ago it was believed that at least some of them were forming stars now for the very first time. Nowadays it is evident that even the most metal-poor ones (like IZw18) contain stars at least 1 Gyr old (Momany et al. 2005). This means that SF should have proceeded for at least a few Gyr in dIrrs, albeit at a low intensity. The low intensity of the SF in DGs is the best way to explain their chemical characteristics, like for instance the low $[\alpha / \mathrm{Fe}]$ ratio. As explained in the introduction, the $[\alpha / \mathrm{Fe}]$ vs. $[\mathrm{Fe} / \mathrm{H}]$ plot is representative of the different delay in production of $\alpha$-elements (mostly produced by the short-living massive stars) and the iron $(2 / 3$ of which comes from longer-living binary systems originating Type Ia SNe). If the SF duration in a galaxy is very short, Type Ia SNe do not have enough time to restore Fe into the ISM and most of the stars will be over-abundant in $\alpha$-elements compared to Fe. The low average $[\alpha / \mathrm{Fe}]$ ratios in dIrrs (compared to large galaxies) is a hint of a long-lasting (presumably very mild) SF in these galaxies (Ikuta \& Arimoto 2002; Lanfranchi \& Matteucci 2004). The same trend of $[\alpha / \mathrm{Fe}]$ (decreasing as we move towards dwarf galaxies) can be obtained by varying the IMF (Recchi et al. 2009), but the hypothesis of a low SF efficiency in dwarf galaxies is important also to explain the downsizing (Cowie et al. 1996), namely the trend of having older stellar populations as we move towards more massive galaxies, indicative of a shorter (but more intense) duration of the SF in large galaxies.

On the other hand, most people believe that a fundamental role in the chemical evolution of dIrrs is played by galactic winds. In fact, these systems are characterized by shallow potential wells and less energy is required to extract gas from them. Galactic winds have the effect of reducing the metallicity of a galaxy in comparison with the one predicted by the closed box model. However, detailed numerical simulations (D'Ercole \& Brighenti 1999; Recchi et al. 2006) have shown that galactic winds are not very 
effective in removing gas from a galaxy. In fact, galactic winds develop vertically (along the direction of steepest pressure gradient) whereas the horizontal transport along the disk (where most of the gas lie) is very limited. On the other hand, the metals freshly produced during a starburst can be easily carried out of the galaxies through a galactic wind (which will be therefore metal-enhanced) and this, too, contributes to keep the metallicity of the galaxy low. Although this overall picture is almost widely accepted, the effect of galactic winds depends very sensibly on parameters like galaxy structure and ISM properties. In particular, the presence of clouds can hamper the development of galactic winds while in the meantime, through evaporation of metal-poor gas, reducing the average metallicity of the galaxy. Detailed numerical simulations (Recchi \& Hensler 2007) show that the leakage of metals is not prevented by the presence of clouds and that the final metallicity is a few tenths of dex lower than in models without clouds.

\section{Concluding Remarks}

Because of its importance for understanding the evolution of baryonic structures in the Universe, their chemical evolution is one of the main focusses of galaxy investigations. For simplicity, numerous analytical approaches have been performed for different morphological units. They can provide a first insight into the temporal and spatial element enrichment by stellar nucleosythesis products.

Since dynamical effects of different gas phases affect the element enrichment differentially leading to a redistributions of those elements, but because also plasmaphysical processes as e.g. heat conduction and turbulence allow for a mixing of gas, more complex dynamically and chemically coupled descriptions have to be developed for advanced models. The development and application of multi-phase so-called chemo-dynamical descriptions are on the way.

How important the implementation of the multi-phase character of the ISM is, can be simply understood by the following basic considerations of its inherent nature: In a low-mass galaxy the stellar energy release can overcome the binding energy of the whole gas reservoir and the gas is totally lost - and by this also the freshly produced metals. Semi-analytical (Salvadori et al. 2008) or single-phase hydrodynamical (MacLow \& Ferrara 1999) models largely overestimate the gas loss due to the instantaneous energy mixture, but to some extent also because of an arbitrarily high SF rate. Although the first-order 1D chemo-dynamical approach (Hensler et al. 2004b) even overestimates the momentum transfer by the drag exerted from hot gas flows to the cool clouds and leads to an overly more efficient gas removal than in 2D or 3D chemo-dynamical models e.g. of dIrrs (Hensler et al. 1999), the model description is still closer to reality because it allows the outflow of hot gas, while hampered by mass loading and drag of cool infalling intergalactic clouds. The amount of expelled metals is reduced (Recchi \& Hensler 2007).

On massive galaxy scales, as e.g. the evolution of giant ellipticals (gE) (Pipino et al. 2006), however, this picture can turn. When hot SNeII gas of $10^{6}-10^{7} \mathrm{~K}$ mixes with a sufficiently high amount of $10^{4} \mathrm{~K}$ and cooler gas, a rough estimate gives that with a SF efficiency of $10 \%$ and a Salpeter IMF about $10 \%$ of the stellar mass are returned by massive star ejecta, i.e. winds + SNeII, what is $1 \%$ of the initial SF gas. Accordingly, the temperature of the mixed gas then amounts to $2.110^{4}-1.210^{5} \mathrm{~K}$, i.e. always in the range of shortest cooling time, and, by this, remains always bound. A collapsing massive galaxy is not stopped and metal-enriched gas is completely following the collapse. Lateron, during the evolution this changes due to the gas consumption so that the SN gas dominates and gas at above $10^{6} \mathrm{~K}$ has reasonably to re-expand (Pipino et al. 2006). It is not surprising that out of a model grid any model will be found to fit the observations 
at best, but nonetheless the validity of its issued parameters must be questioned. Again the simple 1D chemo-dynamical model (Theis, Burkert, \& Hensler 1992) demonstrates that a metallicity of 1-2 times solar can be reached within $1 \mathrm{R}_{e}$ of a gE, while the hot gas with 3-4 times solar metallicity extends out to $10 \mathrm{R}_{e}$.

The here discussed considerations aim at sensibilizing the reader to the validity of $\mathrm{CE}$ models. Finally, we wish to mention that the considerations of CE of tidal-tail DGs, of the intra-cluster medium, of Ly-alpha structures in the early Universe, and furthermore, are also in the focus of crucially important studies and provide further details and to some extent initial and boundary conditions for the CE of galaxies.

Moreover, one should also keep in mind that the stellar yields applied to CE models are still uncertain and change due to advanced stellar evolutionary models, taking into account stellar rotation (see e.g. Chiappini et al. 2003, Hirschi et al. 2005, Meynet et al. 2006), binarity (De Donder \& Vanbeveren 2004), stellar winds, etc.

\section{Acknowledgements}

The authors wish to thank A. Hren for providing some figures, the symposium organizers for their invitation to this review, and the University of Vienna for travel support.

\section{References}

Argast, D., Samland, M., Gerhard, O. E., \& Thielemann, F.-K. 2000, A\&A, 356, 873

Beers, T. C. \& Christlieb, N. 2005, ARAA, 43, 531

Berczik, P., Hensler, G., Theis, C., \& Spurzem, R. 2003, Ap Space Sci., 284, 465

Bouchard, A., Carignan, C., \& Mashenko, S. 2003, AJ, 126, 1295

Burkert, A., Truran, J. S. W., \& Hensler, G. 1992, ApJ, 391, 651

Carigi, L., Peimbert, M., Esteban, C.,\& Garcia-Rojas, J. 2005, ApJ, 623, 213

Carollo, D., Beers, T. C., Lee, Y. S., et al. 2005, Nature, 450, 1020

Cescutti, G., Matteucci, F., McWilliams, A., \& Chiappini, C., 2009 A\&A, 505, 605

Chiappini, C., Matteucci, F., \& Gratton, R. 1997, ApJ, 477, 765

Chiappini, C., Matteucci, F., \& Meyner, G. 2003, A\&A, 410, 257

Colavitti, E., Cescutti, G., Matteucci, F., \& Murante, G. 2009, A\&A, 496, 429

Cowie, L. L., Songalia, A., Hu, E. M., \& Cohen, J. G. 1996, AJ, 112, 839

Dekel, A. \& Woo, J. 2003, MNRAS, 344, 1131

De Donder, E. \& Vanbeveren, D. 2004, New Astron. Rev., 48, 861

D'Ercole, A. \& Brighenti, F. 1999, MNRAS, 309, 941

Eggen, O. J., Lynden-Bell, D., \& Sandage, A. 1962, ApJ, 136, 748

Esteban, C., García-Rojas, J., Peimbert, M., et al. 2005, ApJ (Letters), 618, L95

Ferrini, F., Matteucci, F., Pardi, M. C., \& Penco, U. 1992, ApJ, 387, 138

Ferrini, F., Molla, M., Pardi, M. C., \& Diaz, A. I. 1994, ApJ, 427, 745

Frebel, A., Aoki, W., Christlieb, N., et al. 2005, Nature, 434, 871

Grebel, E. K., Gallagher, J. S., \& Harbeck, D. 2003, AJ, 125, 1966

Harfst, S., Theis, C., \& Hensler, G. 2006, A\&A, 499, 509

Hensler, G. 2003, in: C. Charbonnel et al. (eds.), ASP Conf. Ser. Vol., 304, 371

Hensler, G. 2008, in: J. Andersen, J. Bland-Hawthorn, \& B. Nordstroem (eds.), The Galaxy Disk in Cosmological Context, Proc. IAU Symp., 254, p. 269

Hensler, G., Rieschick A., \& Koeppen, J. 1999, in: J. Beckman \& T.J. Mahoney (eds.), The Evolution of galaxies on Cosmological Timescales, ASP Conf. Ser., 187, 214

Hensler, G., Koeppen, J., Pflamm, J., \& Rieschick, A. 2004a, in: P.-A. Duc, J. Braine, \& E. Brinks (eds.) Recycling intergalactic and interstellar matter, Proc. IAU Symp., 217, 178

Hensler, G., Theis, C., \& Gallagher, J. S., III. 2004b, A\&A, 426, 25

Hirschi, R., Meynet, G., \& Maeder, A. 2005, A\&A, 433, 1013

Ikuta, C. \& Arimoto, N. 2002, $A \& A, 391,55$

Kennicutt, R. C. 1989, ApJ, 344, 685

Knauth, D. C., Meyer, D. M., \& Lauroesch, J. T. 2006, ApJ (Letters), 647, L115 
Koch, A. 2009, $A N, 330,675$

Koeppen, J. \& Hensler, G. 2005, A\& A, 434, 531

Koeppen, J., Theis, Ch., \& Hensler, G. 1998, A\&A, 328, 121

Kroeger, D., Hensler, G., \& Freyer, T. 2006 A\&A (Letters), 450, L5

Lanfranchi, G. A. \& Matteucci, F. 2004, MNRAS, 351, 1338

Maciel, W. J. R., Costa, D. D., \& Uchida, M. M. M. 2003, A\& A, 397, 667

MacLow, M.-M. \& Ferrara, A. 1999, ApJ, 513, 142

Maeder, A. 1992, $A \& A, 264,105$

Marcolini, A., D'Ercole, A., Brighenti, F., \& Recchi, S. 2006, MNRAS, 371, 643

Matteucci, F. 2001, The chemical evolution of the Galaxy, (Kluwer, Dordrecht)

Metz, M., Kroupa, P., Theis, C., Hensler, G., \& Jerjen, H. 2009, ApJ, 697, 269

Meynet, G. \& Maeder, A. 2002a, A\&A (Letters), 381, L25

Meynet, G. \& Maeder, A. 2002b, A\&A, 390, 561

Meynet, G., Ekström, S., \& Maeder, A. 2006, A\&A, 447, 623

Momany, Y., Held, E. V., Saviane, I., et al. 2005, A\&A, 439, 111

Nordstroem, B., Mayor, M., Andersen, J., et al. 2004, A\&A, 418, 989

Oey, M. S. 2000, ApJ (Letters), 524, L25

Pagel B. E. J. 1987, in: G. Gilmore \& B. Carswell (eds.) The Galaxy, (Reidel, Dordrecht), p. 341

Pagel, B. E. J. 1997, Nucleosynthesis and galactic chemical evolution, (Cambridge Univ. Press)

Pagel, B. E. \& Patchett, B. E. 1975, ApJ, 631, 976

Pflamm-Altenburg, J. \& Hensler, G. 2010, MNRAS, submitted

Pipino, A., Matteucchi, F., \& Chiappini, C. 2006, ApJ, 638, 739

Portinari, L. \& Chiosi, C. 1999, A\&A, 350, 837

Portinari, L. \& Chiosi, C. 2000, A\&A, 355, 929

Prantzos, N. 2008a, in: C. Charbonnel \& J.-P. Zahn (eds.), (EDP Sciences) Stellar Nucleosynthesis: 50 years after $B^{2}$ FH; EAS Publication Ser., 32, 311

Prantzos, N. 2008b, A\&A, 489, 525

Recchi, S., Calura, F., \& Kroupa, P. 2009, A\&A, 499, 711

Read, J. I., Wilkinson, M. I., Evans, N. W., et al. 2006, MNRAS, 366, 429

Recchi, S. \& Hensler, G. 2007, A\& A, 476, 841

Recchi, S., Hensler, G., Angeretti, L., \& Matteucci, F. 2006, A\& A, 445, 875

Recchi1, S., Spitoni, E., Matteucci, F., \& Lanfranchi, G. A. 2008, A\& A, 489, 555

Revaz, Y., Jablonka, P., Sawala, T., et al., 2009 A\&A, 501, 189

Salvadori, S., Ferrara, A., \& Schneider, R. 2008, MNRAS, 386, 348

Samland, M. 1994, Ph.D. thesis, Univ. of Kiel, Germany

Samland, M. \& Gerhard, O. 2003, A\& A, 399, 961

Samland, M., Hensler, G., \& Theis, Ch. 1997, ApJ, 476, 544

Sandage, A. \& Fouts, G. 1987, AJ, 93, 74

Scannapieco, C., Tissera, P. B., White, S. D. M., \& Springel, V. 2006, MNRAS, 371, 1125

Schaller, G., Schaerer, D., Meynet, G., \& Maeder, A. 1992 A\&AS, 96, 269

Searle, L. \& Zinn, R. 1978, ApJ, 225, 357

Shaver, P. A., McGee, R. X., Newton, et al. 1983, MNRAS, 204, 53

Spitoni, E., Matteucci, F., Recchi, S., et al. 2009, A\&A, 504, 87

Tadross, A. L. 2003, New Astronomy, 8, 737

Talbot, A. J. \& Arnett, W. D. 1971, ApJ, 170, 403

Theis, C., Burkert, A., \& Hensler, G. 1992, A\&A, 265, 465

Timmes, F. X., Woosley, S. E., \& Weaver, T. A. 1995, ApJS, 98, 617

Tinsley, B. 1974, ApJ, 192, 629

Tinsley, B. 1980, Fund. Cosm. Phys., 5, 287

Tolstoy, E., Hill, V., \& Tosi, M. 2009, ARAA, in press

Twarog, B. A. 1980, ApJ, 242, 242

Venn, K., Irwin, M., Shetrone, M. D., et al. 2004, AJ, 128, 1177

Vilchez, J. M. \& Esteban, C. 1996, MNRAS, 280, 720 